POST COMMON ENVELOPE BINARIES \& EVOLUTION 


\title{
UNDERSTANDING THE LONG-TERM EVOLUTION OF CVS AND LMXBS: PRINCIPLES AND PROBLEMS
}

\author{
U. KOLB \\ Lick Observatory, UCSC, Santa Cruz CA 95064, USA, \\ and Max-Planck-Institut für Astrophysik, \\ Karl-Schwarzschild-Str. 1, 85740 Garching, Germany
}

\begin{abstract}
We introduce the standard model for formation and evolution of $\mathrm{CVs}$, and show how observed collective properties compare with theoretically predicted population models. A short overview relates LMXBs to CVs.
\end{abstract}

\section{Introduction}

The standard model for formation and evolution of cataclysmic variables (CVs) rests on principles formulated decades ago: Kraft, Mathews \& Greenstein (1962) realized the importance of gravitational wave emission for driving mass transfer in close binaries, an idea later refined by Faulkner (1971). Stimulated by Ritter's arguments $(1976 \mathrm{a}, \mathrm{b})$ in favour of a wide binary origin of CVs, with a red giant as progenitor of the white dwarf, Paczyński (1976) suggested a common envelope phase as a mechanism to form a system with an orbital distance much smaller than the radius of the giant once was. Finally Spruit \& Ritter (1983) and Rappaport, Verbunt \& Joss (1983) independently explained the CV period gap by what is now known as the disrupted magnetic braking model. Low-mass X-ray binaries (LMXBs), the neutron star analogues of $\mathrm{CVs}$, were long thought to evolve in a similar way. But although the models reproduce the main features of the observed collective properties of CVs fairly well (Sect. 2) and seem to be incomplete only in detail (Sect. 3), some more fundamental problems remain for LMXBs (Sect. 4).

We restrict this review to Galactic CVs and LMXBs with hydrogen donors. Compact binaries in globular clusters are likely to form via tidal capture and are discussed elsewhere (e.g. Livio 1996; Hut et al. 1992). 


\section{Principles: formation and evolution of CVs}

CVs are thought to form from rather wide main sequence binaries, with an initial separation such that the faster evolving, more massive primary fills its Roche lobe as a giant with predominantly convective envelope. The resulting mass transfer is dynamically unstable and leads to a common envelope (CE) phase, during which orbital energy is used to expel the giant's envelope on a very short time-scale. Left behind in a very close orbit are the exposed core, the later white dwarf (WD), and the almost unaffected lowmass, main sequence secondary. Loss of orbital angular momentum by magnetic stellar wind braking and gravitational wave emission, or slow nuclear expansion of the secondary, establish the semi-detached CV configuration. These angular momentum losses maintain continuous mass transfer from the secondary at a rate $\dot{M} \simeq 10^{-9} \ldots 10^{-8} \mathrm{M}_{\odot} \mathrm{yr}^{-1}$ for long orbital period, $P$, and $\dot{M} \simeq 10^{-11} \ldots 10^{-10} \mathrm{M}_{\odot} \mathrm{yr}^{-1}$ for short $P$, where only gravitational radiation operates. As a reaction to mass loss the secondary builds up a gravo-thermal luminosity, causing the stellar radius to become smaller (for a secondary mass $\left.M_{2} \gtrsim 0.6 \mathrm{M}_{\odot}\right)$ or larger $\left(M_{2} \lesssim 0.6 \mathrm{M}_{\odot}\right)$ than the main sequence radius. With progressing mass transfer the secondary's outer convection zone penetrates deeper into the star, until at $M_{2} \simeq 0.2 \mathrm{M}_{\odot}$ the donor becomes fully convective and magnetic braking is assumed to vanish discontinuously. The system detaches since the secondary's contraction on a thermal time-scale is faster than the orbital shrinking on the much longer gravitational radiation time-scale. When mass transfer resumes the secondary has regained thermal equilibrium and the orbital period is correspondingly smaller. Systems in the detached phase are not observable as $\mathrm{CV}_{\mathrm{s}}$, they are missing in the orbital period distribution of CVs and cause the well-known $2 \ldots 3 \mathrm{~h}$ period gap. The donor eventually becomes a degenerate brown dwarf, causing $P$ to increase, even though the orbital angular momentum further decreases. Accordingly, the system passes through a minimum orbital period $P_{\min }$ at $\simeq 80 \mathrm{~m}$. Further details of this evolutionary scheme are given by e.g. King (1988) and Kolb (1995b).

Along these lines detailed population synthesis calculations try to link observed properties of newly born main sequence binaries (the star formation rate, the distribution of primary mass, mass ratio and orbital separation) to observed collective properties of CVs like the CV period distribution by applying results from stellar evolution theory. Explicit computations of the CV formation rate were carried out by Politano (1988, 1996) and de Kool (1992). Kolb (1993) combined these models with detailed evolutionary sequences of CVs to obtain a multi-dimensional distribution function of system parameters representing the present Galactic CV population. Other, more extended, binary population synthesis calculations 
do not consider CVs in detail (e.g. Han, Podsiadlowski \& Eggleton 1995; Yungelson et al. 1995).

Here we summarize the main results of $\mathrm{CV}$ population studies; for basic concepts and interpretations see Kolb (1995b):

Main sequence binaries qualify as CV progenitors if (i) the primary can form a WD core within the age of the Galaxy but avoids a supernova (primary mass $0.8 \lesssim M_{\mathrm{p}} / \mathrm{M}_{\odot} \lesssim 9$ ) and (ii) the mass ratio $q_{\mathrm{i}}=M_{\mathrm{p}} / M_{2}$ is large ( $~ Z 3.5$ ), so that mass transfer is stable when the post-CE binary becomes semi-detached. Roughly, about 30 out of 100 main sequence binaries within the above mass range encounter dynamically unstable mass transfer leading to a CE. About 10 binaries survive the CE, 5 close enough to turn on mass transfer within a Hubble time, but only 2 in a stable configuration as a $\mathrm{CV}$. Depending on the initial masses the possible initial separation range is $100 \ldots 1200 \mathrm{R}_{\odot}($ period range $\simeq 60 \ldots 2500 \mathrm{~d})$. The predicted $\mathrm{CV}$ formation rate depends most sensitively on the initial mass ratio distribution $g\left(q_{\mathrm{i}}\right)$. Calibrated to the observed formation rate of isolated WDs the binary population models find for the number of CVs residing in the Galaxy today about $210^{6}$ [for $g\left(q_{\mathrm{i}}\right) \propto q_{\mathrm{i}}^{-3}$ ] to $2010^{6}$ (for uncorrelated masses), i.e. an intrinsic CV space density $10^{-5} \ldots 10^{-4} \mathrm{pc}^{-3}$.

To estimate the post $-\mathrm{CE}$ configuration usually a constant value $\leq 1$ is assumed for the fraction, $\alpha_{\mathrm{CE}}$, of released orbital binding energy available to unbind the envelope. Given the complexity of the CE phase (see e.g. Iben \& Livio 1993) it is not surprising that more recent numerical simulations cast some doubt on this concept (e.g. Yorke, Bodenheimer \& Taam 1995; Terman \& Taam 1996). Unless $\alpha_{\mathrm{CE}}$ is a strong function of pre CV binary parameters this should have no significant effect on synthesis results for CVs which are not very sensitive to the actual global value of $\alpha_{\mathrm{CE}}$.

Fig. 1 (left) depicts the period distribution of a visual magnitude ( $\left.m_{\text {vis }}\right)$ limited sample obtained from one of the intrinsic population models by Kolb (1993; $\alpha_{\mathrm{CE}}=1$, uncorrelated masses, magnetic braking according to Verbunt \& Zwaan 1981). The system's visual magnitude $L_{\text {vis }}$ is assumed to be that of a stationary accretion disk and can be estimated by $L_{\mathrm{vis}} \propto M_{1}^{\alpha} \dot{M}^{\beta}$, where $M_{1}$ is the WD mass, and $\alpha \simeq 1, \beta \simeq 3 / 4$ (Dünhuber 1994). Although intrinsically only $0.5 \%$ of all CVs have periods above the gap (and $\lesssim 70 \%$ have evolved beyond the minimum period), the $m_{\mathrm{vis}}$-limited sample is characterized by roughly an equal number of systems below and above the gap, just as the observed sample (Fig. 1, right). The minimum period in the computed distribution is shorter than $80 \mathrm{~m}$ due to the neglect of rotational and tidal corrections in the stellar models. The spike at $\log P / \mathrm{h} \simeq 0.02$ (Fig. 1, left) results from systems accumulating at $P_{\min }$ where $P=0$. The fact that it is absent in the observed sample points to additional selection effects not accounted for in the model, e.g. the very long dwarf nova recur- 

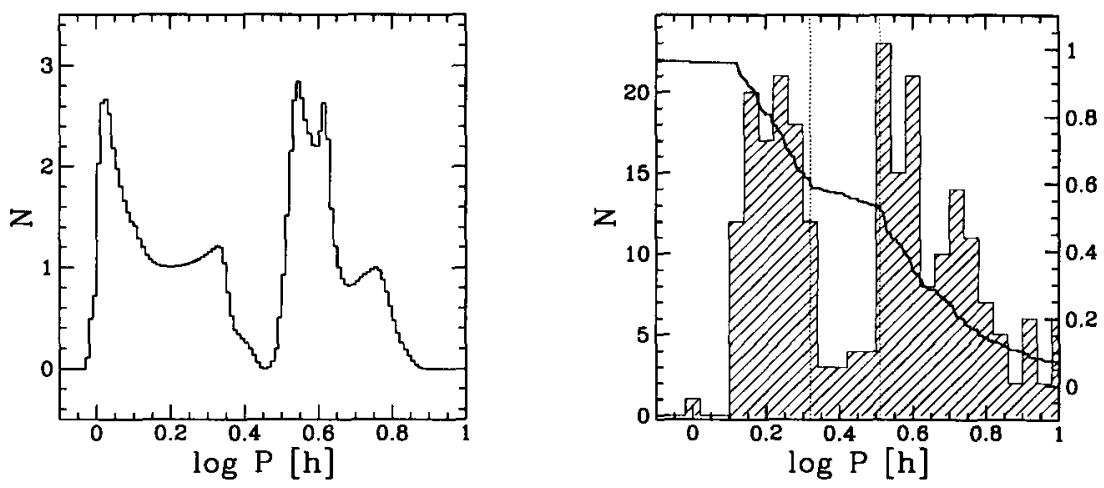

Figure 1. Left: $m_{\text {vis }}$-limited sample obtained from a CV population model by Kolb (1993). Right: Observed CV period histogram (hatched; scale on the left) and cumulative period distribution (scale on the right); data from Ritter (1995). The vertical dotted lines mark the period gap.

rence time at small $P$. The local minimum at $\log P / \mathrm{h} \simeq 0.67$ (Fig. 1, left) is due to an allowance for the formation of $\mathrm{H}_{2}$ molecules in the outer layers of the secondary and should eventually show up in an observed sample with improved statistics, whereas the spike at $\log P / \mathrm{h} \simeq 0.62$ is just a numerical artefact (see Kolb 1993).

\section{Problems: the period distribution of subtypes}

The standard model introduced in the previous section seems to agree fairly well with the main features of the observed CV population, but a closer look at various CV subtypes reveals properties the models, as discussed so far, fail to reproduce.

Dwarf novae and nova-likes. Observations indicate that above the period gap the relative number of dwarf novae per period bin is an increasing function of orbital period (Fig. 2, left). In contrast, standard evolutionary models combined with predictions from the disk instability model for dwarf nova outbursts (see e.g. Cannizzo 1993 for a review) tend to predict the opposite. As an example, Fig. 2 (right) depicts different evolutionary sequences in the $P-\dot{M}$ plane, together with a limiting line separating stable (nova-likes, above the line) from unstable (dwarf novae, below) systems. The line specifies the location of CVs with a disk just cool enough for hydrogen recombination at its outer edge. Even though the precise run of $\dot{M}$ with $P$ in $\mathrm{CVs}$ depends on the detailed mechanism extracting angular 

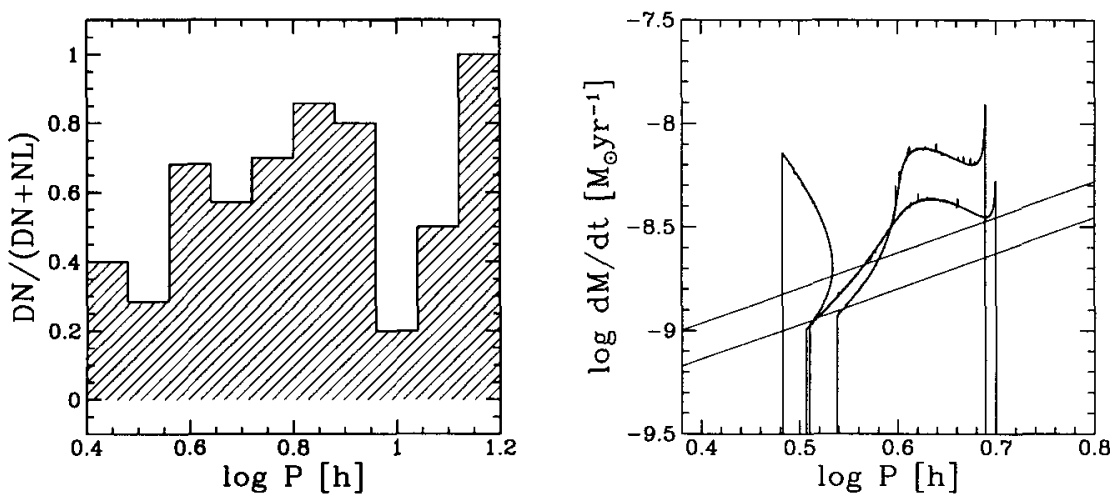

Figure 2. Left: The dwarf nova fraction in non-magnetic CVs above the period gap as a function of orbital period. Data from Ritter (1995). Right: Mass transfer rate versus orbital period for various evolutionary tracks of CVs (taken from Kolb \& Ritter 1992). The initial WD/secondary masses (in $\left.\mathrm{M}_{\odot}\right)$ are $(1.0,0.6),(0.7,0.6)$ and $(1.0,0.4)$, in order of decreasing turn-on period. The straight lines denote the critical mass transfer rate for DN outbursts for a disk radius of 0.7 (upper line) and 0.6 (lower line) times the WD's Roche lobe radius (following Shafter 1992).

momentum from the system, the mass transfer rate at the upper edge of the period gap can be deduced in a model-independent way from the observed width of the gap: The gap width measures the ratio $r=R_{2} / R_{\mathrm{e}}$ of the radius of the secondary when it enters $\left(R_{2}\right)$ and leaves $\left(R_{\mathrm{e}}\right)$ the detached phase. Since $R_{\mathrm{e}}$ is the thermal equilibrium radius, the value of $r$ can be related to the ratio $\tau=t_{\mathrm{KH}} / t_{\mathrm{M}}$ of Kelvin-Helmholtz time-scale, $t_{\mathrm{KH}}$, and mass loss time-scale, $t_{\mathrm{M}}$ (Stehle, Ritter \& Kolb 1996), at the upper edge of the gap. Hence the mass transfer rate results from $\tau$ and the mass of the secondary in the detached phase, known from the location of the lower edge of the gap. The observed gap between $2.09 \mathrm{~h}$ and $3.24 \mathrm{~h}$ (limits taken from the cumulative period distribution shown in Fig. 1, right) requires $r \simeq 4 / 3$ and $\tau \simeq 5$, so that $\dot{M} \simeq 10^{-9} \mathrm{M}_{\odot} \mathrm{yr}^{-1}$. This value is below the critical limit for disk instability, even for an unusually small disk (see Fig. 2). Therefore close to the upper edge of the gap virtually all systems should have unstable disks, whereas only a few dwarf novae exist in this period regime, a fact already noted by Shafter (1992).

A related problem is the apparent scatter of the observed mass transfer rate at a given orbital period (e.g. Patterson 1984, Warner 1987) which seems to be at least an order of magnitude larger than the standard model can account for. Since the reaction of low-mass secondaries to mass loss demands a fairly uniform evolution (Stehle et al. 1996) this scatter was interpreted as a cyclic change of the instantaneous mass transfer rate around 
the secular mean, on a time-scale too long to be observable directly, but short enough in order not to change the secular mean (e.g. Hameury, King \& Lasota 1989). Although weak irradiation of the secondary by the WD was identified as a promising mechanism causing such cycles (King et al. 1995a; Ritter et al. 1996a,b), a more recent model-independent analysis shows that purely irradiation driven cycles are possible only for $M_{2} \gtrsim 0.7 \mathrm{M}_{\odot}$ (Ritter, Zhang \& Hameury 1996; King et al. 1995b). Additional mechanisms, e.g. a mass transfer dependent (consequential) angular momentum loss component (King \& Kolb 1995), are neecied to allow mass transfer cycles at periods close to the upper edge of the gap. These in turn could help to resolve the problem of the dwarf nova period distribution.

Systems in the period gap. As listed in Table 1, 19 systems out of a total number of $269 \mathrm{CVs}$ have orbital periods in the period gap range $2.09 \ldots 3.24 \mathrm{~h}$ (data from Ritter 1995). Apparently there is an overabundance of polars and - although this is clearly small number statistics classical novae in the gap. Possible implications for the influence of the WD magnetic field on the long-term evolution were reviewed recently by several authors (King 1994; Chanmugam 1995; Kolb 1995b) and will not be repeated here. A gap population of $\simeq 7 \%$ is significantly above the prediction $\lesssim 3 \%$ from computed $m_{\text {vis }}$-limited samples such as the one shown in Fig. 1, but the observed gap population of non-magnetic CVs is still at least marginally consistent with the models. For classical novae see below.

TABLE 1. CVs in the period gap

\begin{tabular}{llll}
\hline Subtype & $\begin{array}{l}\text { systems } \\
\text { in gap }\end{array}$ & $\begin{array}{l}\text { \% of } \\
\text { gap population }\end{array}$ & $\begin{array}{l}\text { \% of CVs with known } P \\
\text { belonging to subtype }\end{array}$ \\
\hline non-magnetic dwarf novae & 5 & 26 & 41 \\
non-magnetic nova-likes & 2 & 10 & 15 \\
VY Scl systems & 1 & 5 & 5 \\
polars & 6 & 32 & 16 \\
intermediate polars & 2 & 10 & 9 \\
classical novae & 3 & 16 & 8 \\
\hline
\end{tabular}

Classical novae. A nova occurs whenever the hydrogen-rich envelope accreted on the WD ignites $\mathrm{H}$-burning in a thermonuclear runaway (TNR). CV population models can be used to predict the distribution of the nova outburst frequency $\nu=\dot{M} / \Delta M_{\text {ign }}$ over system parameters, provided the envelope mass at ignition $\Delta M_{\text {ign }}$ is known. Roughly, the TNR begins when the pressure at the base of the envelope exceeds a critical value (e.g. Livio 
1994). Correspondingly, the nova rate is large for high-mass WD CVs: $\Delta M_{\mathrm{ign}} \propto R_{1}^{4} M_{1}^{-1} \rightarrow 0$ when $M_{1}$ approaches the Chandrasekhar limit. The main character of this dependency remains the same when results from an extended grid of more detailed TNR model computations, applying a diffusion description for envelope/WD material mixture prior to ignition (Prialnik \& Kovetz 1995), replace this simple ignition criterion (Kolb 1995a). As an immediate consequence the mean WD mass obtained from the WD mass distribution of $\nu$ is very large, typically $\left\langle M_{1}\right\rangle \simeq 1.3 \mathrm{M}_{\odot}$, whereas observed values, though subject to considerable uncertainty, point to a much smaller value $\left(\simeq 0.8 \mathrm{M}_{\odot}\right)$. The period distribution of $\nu$ is very similar to the $m_{\text {vis }}$-limited sample computed for CVs (Fig. 1) since in both cases the intrinsic distribution is weighted with a selection factor $\propto \dot{M}$. In particular, a period gap is predicted and the expected fraction of novae below the gap is typically of order $50 \%$.

This is in marked contrast to the distribution of the small sample of 21 novae with determined orbital period (Fig. 3, left): 3 novae fall below the classical CV period gap, 3 within the gap (see Table 1), so that the observed distribution shows no signature of a gap at all (whereas polars do show a gap, see e.g. Kolb 1995b). It is not clear if this is due to small number statistics, selection effects operating against the detection of novae in systems with small mass transfer rate $\lesssim 10^{-10} \mathrm{M}_{\odot} \mathrm{yr}^{-1}$, or differences in the outburst behaviour of high $-\dot{M}$ and low $-\dot{M}$ novae. Alternatively, a closer inspection of the relation between mass transfer cycles and nova outbursts and the role of dynamical friction when the secondary orbits in the expanding nova envelope (see Schenker, Kolb \& Ritter 1996 for further references) might resolve some of these problems.

On the other hand, the total Galactic nova rate predicted from the models, $1 \ldots 10 \mathrm{yr}^{-1}$, is surprisingly close to the presently favoured observed value (20 $\mathrm{yr}^{-1}$, Della Valle \& Livio 1994; but see also Della Valle \& Duerbeck 1993).

\section{Relatives: low-mass X-ray binaries}

The purpose of this final section is to emphasize the difference between evolutionary histories of LMXBs and CVs. No attempt is made to provide a review on formation and evolution of LMXBs (for which the reader is referred to e.g. Bhattacharya \& van den Heuvel 1991; Verbunt 1993; D'Antona 1996).

The main additional obstacle for the formation of LMXBs is that the progenitor binary has to survive the supernova explosion required to produce a neutron star. The corresponding constraints on the initial parameter space limit the LMXB formation rate to a value well below the CV birth 

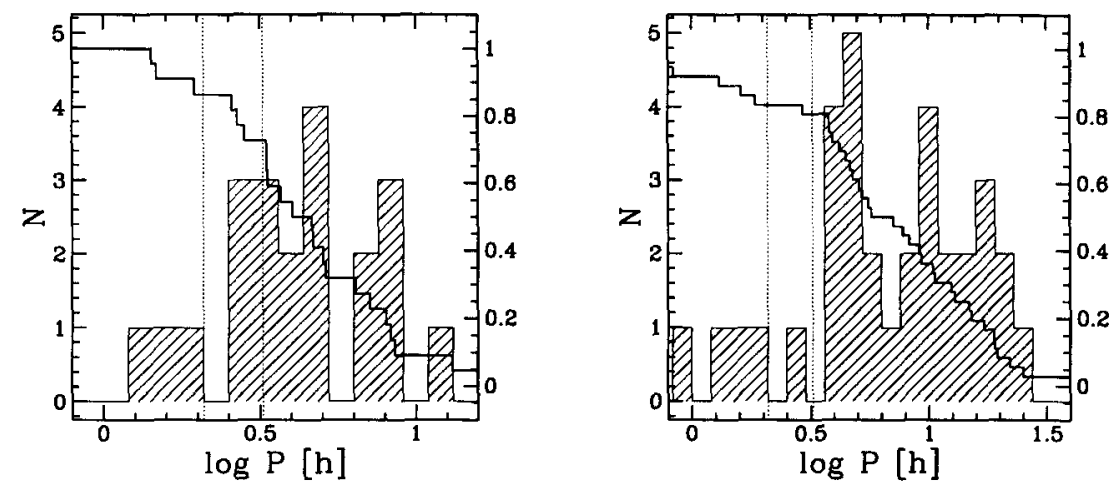

Figure 3. Observed period histogram (hatched; scale on the left) and cumulative period distribution (scale on the right) for classical novae (left) and low-mass X-ray binaries (right). Data from Ritter (1995).

rate.

A possible LMXB producing evolutionary channel very similar to the $\mathrm{CV}$ case is sometimes referred to as the standard model: here the initially very massive primary $\left(10 \lesssim M_{\mathrm{p}} / \mathrm{M}_{\odot} \lesssim 50\right)$ emerges from the orbitshrinking CE phase as a helium star. Subsequently it passes through a Wolf-Rayet phase and explodes finally as a supernova (SN). For spherically symmetric mass ejection the binary remains bound only if less than half of the pre-SN mass leaves the system. Since this is typically not the case (the He star generally being more massive than its companion) many authors invoke kick velocities to circumvent the disruption of the binary. The still-detached post-SN binary could be observable as a binary radio pulsar if the neutron star were magnetic.

More exotic ways to generate LMXBs have been suggested, e.g. a triple star origin (Eggleton \& Verbunt 1986), SN kicks causing small orbits without involving a CE (Kalogera 1996), or accretion induced collapse of a massive WD to produce the neutron star in an already existing close binary, thus avoiding major mass loss (Whelan \& Iben 1973).

Once a LMXB is formed its long-term evolution should be determined by the same principles as for CVs. However, the LMXB orbital period distribution (see Fig. 3, right) extends to longer periods, i.e. the fraction of binaries with (sub)giant donors is significantly larger than for CVs, and there are only few systems below $P \simeq 4 \mathrm{~h}$. Accordingly, no well-defined period gap or minimum period exists. More indirect evidence for differences from a standard 'CV-type' evolution comes from estimates for the formation rate of low-mass binary pulsars, which are believed to descend from 
LMXBs. Kulkarni \& Narayan (1988) found a value too large by a factor $10 \ldots 100$ to be compatible with a characteristic LMXB lifetime of $10^{9} \mathrm{yr}$. However, more recent estimates (Johnston \& Bailes 1991) suggest a much more moderate discrepancy.

To explain these findings it was pointed out that irradiation of the secondary by either the $\mathrm{X}$-ray radiation from the vicinity of the neutron star during the LMXB phase or pulsar radiation during a detached state might alter the long-term evolution significantly. Two model categories can be distinguished, both potentially leading to an accelerated evolution: the first group considers the effect X-ray heating has on the secondary's internal structure. Deposited below the photosphere the incoming flux blocks internally generated luminosity, thereby expanding the donor star and driving an elevated mass transfer rate (Podsiadlowski 1991). The main unknown here is the time-scale and amplitude of the expansion, determined essentially by how efficiently the star can cool through the unirradiated side (see e.g. Hameury et al. 1993). The second group considers evaporation of the star's atmospheric layers, i.e. substantial mass loss in a strong stellar wind (e.g. Tavani 1991). Again, the quantitative implications remain speculative.

In conclusion, both for the understanding of details in the observed inter-relation of CV subtypes and of the fundamental evolution of LMXBs more investigations considering effects of weak and strong irradiation on (nearly) Roche lobe filling low-mass stars are urgently needed.

Acknowledgements. I would like to thank R. Taam and J. Frank for stimulating conversations, and J. Faulkner for comments and improving the language of the manuscript. Support from an IAU travel grant and NASA grant NAGW 3599 is greatly acknowledged.

\section{References}

Bhattacharya, D., van den Heuvel, E. P. J., 1991, Phys. Rep., 203, 1

Cannizzo, J. K., 1993, in "Accretion disks in compact stellar systems", ed. J. C. Wheeler, World Scientific, p6

Chanmugam, G. 1995, in "Cataclysmic Variables", eds A. Bianchini et al., Kluwer, p415

D'Antona, F., 1996, in "Evolutionary Processes in Binary Stars", eds R. A. M. J. Wijers, M. B. Davies, C. A. Tout, NATO ASI Series, Kluwer, Dordrecht, in press

Della Valle, M., Duerbeck, H., 1993, A\&A, 271, 175

Della Valle, M., Livio, M., 1994, Ap. J., 423, 31

Dünhuber, H., 1994, Ph.D. thesis, Universität München

Eggleton, P. P., Verbunt, F., 1986, MNRAS, 220, 13P

Faulkner, J., 1971, Ap. J., 170, L99

Hameury, J.-M., King, A. R., Lasota, J.-P., 1989, MNRAS, 237, 39

Hameury, J.-M., King, A. R., Lasota, J.-P., Raison, F., 1993, A\&A, 277, 81

Han, Z., Podsiadlowski, P., Eggleton, P. P., 1995, MNRAS, 272, 800

Hut, P., McMillan, S., Goodman, J., et al., 1992, PASP, 104, 981

Iben, I., Livio, M., 1993, PASP, 105, 1373 
Johnston, S., Bailes, M., 1991, MNRAS, 252, 277

Kalogera, V., 1996, in "Proceedings of the $2^{\text {nd }}$ Hellenistic Astronomical Conference", Thessaloniki, Greece, in press

King, A. R., 1988, QJRAS, 29, 1

King, A. R., Kolb, U., 1995, Ap. J., 439, 330

King, A. R., Frank, J., Kolb, U., Ritter, H., 1995a, Ap. J., 444, L37

King, A. R., Frank, J., Kolb, U., Ritter, H., 1995b, Ap. J., submitted

Kolb, U. 1995a, in "Cataclysmic Variables", eds A. Bianchini et al., Kluwer, p511

Kolb, U. 1995b, in "Magnetic Cataclysmic Variables", eds D. Buckley, B. Warner, ASP Conf. Ser. 85, p440

Kolb, U., 1993, A\&A, 271, 149

Kolb, U., Ritter, H., 1992, A\&A, 254, 213

de Kool, M., 1992, A\&A, 261, 188

Kraft, R. P., Mathews, J., Greenstein, J. L., 1962, Ap. J., 136, 312

Kulkarni, S. R., Narayan, R., 1988, Ap. J., 335, 755

Livio, M., 1994, in "Interacting Binaries", eds S. N. Shore, M. Livio, E. P. J. van den Heuvel, Springer, Berlin, p135

Livio, M., 1996, in "Binary Stars in Clusters", ed. E. Milone, ASP Conf. Series, in press

Paczyński, B., 1976, in "Structure and evolution of close binary systems", eds P. Eggleton, S. Mitton, J. Whelan, Reidel, Dordrecht, p75

Patterson, J., 1984, Ap. J. Supp., 54, 443

Podsiadlowski, P., 1991, Nature, 350, 136

Politano, M., 1988, Ph.D. thesis, University of Illinois, Urbana

Politano, M., 1996, Ap. J., in press

Prialnik, D., Kovetz, A., 1995, Ap. J., 445, 789

Rappaport, S., Verbunt, F., Joss, P. C., 1983, Ap. J., 275, 713

Ritter, H., 1976a, in "Structure and evolution of close binary systems", eds P. Eggleton, S. Mitton, J. Whelan, Reidel, Dordrecht, p205

Ritter, H., 1976b, MNRAS, 175, 279

Ritter, H., 1990, A\&AS, 85, 1179

Ritter, H., 1995, A compilation of cataclysmic binaries and related objects with known or suspected orbital periods, version of June 1st, 1995, private communication; last published version: Ritter \& Kolb 1995; Ritter 1990

Ritter, H., Kolb, U., 1995, in "X-ray Binaries", eds W. H. G. Lewin, J. van Paradijs, E. P. J. van den Heuvel, Cambridge University Press, p578

Ritter, H., Zhang, Z., Hameury, J.-M., 1996, these proceedings, p449

Ritter, H., Zhang, Z., Kolb, U., 1996a, in "Compact Stars in Binaries", IAU Symposium 165 , ed E. P. J. van den Heuvel, in press

Ritter, H., Zhang, Z., Kolb, U., 1996b, in preparation

Schenker, K., Kolb, U., Ritter, H., 1996, these proceedings, p447

Shafter, A. W., 1992, Ap. J., 394, 268

Spruit, H. C., Ritter, H., 1983, A\&A, 124, 267

Stehle, R., Ritter, H., Kolb, U., 1996, MNRAS, in press

Tavani, M., 1991, Ap. J. Lett., 366, L27

Terman, J. L., Taam, R. E., 1996, Ap. J., in press

Verbunt, F., 1993, ARA\&A, 31, 93

Verbunt, F., Zwaan, C., 1981, A\&A, 100, L7

Warner, B., 1987, MNRAS, 227, 23

Whelan, J., Iben, I., 1973, Ap. J., 186, 1007

Yorke, H. W., Bodenheimer, P., Taam, R. E., 1995, Ap. J., 451, 308

Yungelson, L. R., Livio, M., Tutukov, A. Kenyon, S. J., 1995, Ap. J., 447, 656 